\title{
Enhancing Contention Resolution ALOHA using Combining Techniques
}

\author{
Federico Clazzer, Student Member, IEEE, Christian Kissling, Mario Marchese, Senior Member, IEEE
}

\begin{abstract}
Recently, random access protocols have acquired a new wave of interest, not only from the satellite communication community, but also from researchers active in fields like Internet of Things and machine-to-machine. Asynchronous (slot- and frame-wise) ALOHA-like random access protocols, are very attractive for such applications, enabling low complexity transmitters and avoiding time synchronization requirements. Evolutions of ALOHA employ time diversity through proactive replication of packets, but the time diversity is not fully exploited at the receiver. Combining techniques, as selection combining and maximal-ratio combining, are beneficial and are adopted in the enhanced contention resolution ALOHA (ECRA) scheme, presented here. A tight approximation of the packet loss rate for asynchronous random access, including ECRA, well suited for the low channel load region is derived. Finally, ECRA is evaluated in terms of spectral efficiency, throughput and packet loss rate in comparison with recent protocols, showing that it is able to largely outperform both slotted synchronous and asynchronous schemes.
\end{abstract}

Index Terms-Diversity methods, Multiaccess communication, Satellite communication, Radio communication, Communication.

\section{INTRODUCTION}

$\mathbf{F}$ IXED allocation, for multiple-access wireless communication systems that are dynamic in terms of resource requests from the transmitting nodes, is normally inefficient. On demand resource allocation, e.g. demand assigned multiple access (DAMA), can be beneficial [1]. However, there are a number of scenarios where DAMA is unable to counteract efficiently the dynamics of the resource requests. For example, when the channel traffic shows a bursty and unpredictable nature, when there is a very high number of transmitters and coordination is hard to achieve, or when delay-critical applications are considered. The last case is of particular importance in geostationary orbit (GEO) satellite communication systems. Before the resource request of a given transmitter can be satisfied, an additional delay of one round trip time of ca. 500 ms may elapse. Such additional delay is critical for several applications [2].

Random access (RA) protocols have evolved significantly from the original idea of ALOHA proposed by Abramson

The material in this paper was presented in part at the 2013 International ITG Conference on Systems, Communications and Coding (SCC).

Federico Clazzer is with the Institute of Communications and Navigation of the German Aerospace Center (DLR), Muenchner Strasse 20, 82234 Wessling, Germany. Email: Federico.Clazzeredlr.de.

Christian Kissling is with the Department for Electrical Engineering and Information Technology of the Munich University of Applied Sciences, Lothstr. 64, 80335 Munich, Germany. E-mail: Christian.Kissling@hm.edu.

Mario Marchese is with the DITEN, University of Genoa, Via Opera Pia 13, 16145 Genova, Italy. E-mail: Mario. Marchese@unige.it. in 1970 [3], and its time slotted evolution [4], [5]. Recently, driven by a number of applications like underwater networks [6], RFID communication systems [7], vehicular ad hoc networks [8], machine-to-machine (M2M) communication systems [9] and satellite networks [10], numerous new RA schemes have been proposed. Among them, worth to be mentioned is contention resolution diversity slotted ALOHA (CRDSA) [11]. The scheme is an evolution of diversity slotted ALOHA (DSA) [12]. With respect to slotted ALOHA (SA), in DSA, lower delays and higher throughput are provided under very moderate channel load conditions, by transmitting in a proactive way two or more times the same packet. The packet instances (replicas in the following) are sent separately with a random delay. Nonetheless, the increase of channel occupation, due to the presence of replicas, is counterproductive for medium channel load conditions, and worsen both throughput and packet loss rate (PLR) performance w.r.t. SA. In DSA arises the tradeoff between time diversity introduced by the presence of replicas, and the channel load increase. When the channel load is limited, replicating packets is beneficial because the collision probability remains relatively low while the probability that at least one replica is received collision-free is higher. Instead, at moderate channel load, the PLR is driven by collisions, and sending replicas harms the performance.

A larger gain is achieved when both time diversity and successive interference cancellation (SIC) are exploited [11]. The CRDSA scheme [11] follows the idea of DSA to send more than one replica per user, and additionally introduces SIC at the receiver. Transmissions are organized into frames, where users are allowed to transmit only once. The users replicate their packets two (or more) times, and place the replicas in slots selected uniformly at random, providing in all replicas the information on the selected slots. At the receiver, SIC exploits the presence of multiple replicas per user for clearing up collisions. Every time a packet is decoded, SIC reconstructs the waveform and subtracts it from all the slot locations selected for transmission by the corresponding user, possibly removing the interference contribution with respect to other packets. The performance evaluations in [11] have shown that the maximum throughput of CRDSA can be impressively extended from $S \cong 0.36$ (the peak throughput of $\mathrm{SA}$, measured in average number of successful transmissions per transmission period [13] or packets per $\left.\operatorname{slot}^{1}\right)$, up to

${ }^{1}$ Following the definition of [13], we assume that a transmission period is equal to $T_{p}$ seconds, which coincides with the physical layer packet duration and also coincides with the slot duration. Therefore, for slotted protocols, the throughput can be measured also in packets/slot. 
$\mathrm{S} \cong 0.55$. For a target PLR of $10^{-3}$, CRDSA can support up to 1 [bits/symbol], considering quadrature phase-shift keying (QPSK) modulation and the 3GPP Turbo Code of rate $1 / 3$ [14]. Further performance improvements can be achieved when, 1) more than two replicas per user and per frame are sent, 2) difference in received power, due to induced power unbalance or fading, and capture effect are considered [14]. The stability of CRDSA has been investigated in [15], while more recently an analytical framework for slotted RA protocols embracing SA, DSA and CRDSA has been presented in [14]. Irregular repetition slotted ALOHA (IRSA) [16] is an extension of CRDSA, where the number of replicas sent by users is drawn from a probability mass function optimized for maximizing the throughput. The bipartite-graph representation is introduced, and exploited for characterizing the interference cancellation (IC) process, helping the optimization procedure, and building a bridge towards tools typically deployed in coding theory. An extension of IRSA, named coded slotted ALOHA (CSA) has been presented and analysed in [17], where the replicas are not simply repetitions of the original packet as in IRSA and CRDSA, but instead, they are coded versions of them. IRSA approaches a theoretical throughput of $S=0.97$ with a distribution containing a maximum of 16 replicas per user, obtained via differential evolution [16]. Both IRSA and CSA are able to achieve a throughput arbitrarily close to 1 packet per slot [18], under the collision channel model [19], letting both the number of slots in the frame, and the maximum number of replicas sent by each user, grow very large. The authors in [20], bridge soft combining with SIC for the CRDSA scheme, showing remarkable gains. In [21] it has been shown that joint decoding of the collided packets can be attempted, resorting to multiuser detection (MUD) techniques. The authors of [9], [22] elaborate the concept of frameless slotted scheme, i.e. the duration of a frame is not a-priori fixed, but the contention ends when the throughput is maximized. Further evolutions of RA include the extension to multiple receiver scenarios [23], [24] and to all-to-all broadcast transmission [25].

Similarly, SIC can also be adopted when considering asynchronous spread spectrum random access, as in [26]. The enhanced spread spectrum ALOHA (E-SSA) uses direct sequence spread spectrum at the transmitter without replicas, i.e. each terminal sends only one packet per transmission. At the receiver side, SIC is employed for removing interference, once packets are correctly decoded. The excellent performance of E-SSA are shown in [27].

For asynchronous RA without spreading, contention resolution ALOHA (CRA) [2] has been the first attempt to mimic the improvements given by CRDSA. Time slots are removed, but frames are kept, and users are allowed to transmit their replicas within the frame, without any constraint excepts avoiding self-interference. At the receiver, SIC is employed to improve the performance, similarly to the slotted counterpart CRDSA. Recently asynchronous contention resolution diversity ALOHA (ACRDA) [28] has removed also the frame structure still present in CRA, reducing once more the transmitter complexity. However, both CRA and ACRDA do not exploit the inherent time diversity of the interference among replicas, which naturally arises due to the asynchronous nature of the protocol, i.e. different portion of replicas of a given user might be interfered.

Driven by this observation, the present paper introduces the enhanced contention resolution ALOHA (ECRA) slot- and frame-wise asynchronous RA scheme. It employs combining techniques in order to resolve collision patterns where SIC alone is unable to succeed. The main contributions of the present work can be summarized as:

- Extension of asynchronous RA protocols towards combining techniques such as selection combining ( $\mathrm{SC}$ ), equalgain combining (EGC) and maximal-ratio combining (MRC) [29], [30]. The novel ECRA exploits time diversity of the interference pattern suffered by the replicas, for creating a combined observation at the receiver, on which decoding is attempted.

- Development of an analytical approximation of the PLR performance, for asynchronous $R A$ schemes, particularly tight for low channel load. The approximation focuses on a subset of collision patterns, unresolvable with SIC. The PLR analytical approximation for ECRA with MRC focuses on the case with two replicas only.

- Comparison of ECRA with asynchronous and slot synchronous protocols under several metrics as throughput, spectral efficiency and normalized capacity.

The reminder of the paper is organized as follows. In Section II, the system model is presented, including the detailed description of the ECRA decoding algorithm. In Section III, the PLR approximation is derived for asynchronous schemes, including ECRA. In Section IV, the performance metrics are defined, and numerical results for ECRA as well as comparison with recent slot synchronous and asynchronous schemes are shown. Finally, in Section V, concluding remarks close the paper.

\section{SySTEM MODEL}

We assume an infinite user population generating traffic, following a Poisson process of intensity G. The channel load ${ }^{2}$ $\mathrm{G}$ is measured in packet arrivals per packet duration $T_{p}$. Upon arrival, each user replicates its packet $d$ times, with $d$ the repetition degree of the system. The first replica is transmitted immediately, while the remaining $d-1$ are sent within a virtual frame $(\mathrm{VF})$ of duration $T_{f}$, starting at the beginning of the first replica. ${ }^{3}$ As a consequence, virtual frames are asynchronous among users. Replicas are sent such that selfinterference is avoided. The time location within the VF of each replica is stored in a dedicated portion of the packet header. Each replica is composed by $k$ information bits. In order to protect the packets against channel impairments and interference, a channel code $\mathcal{C}$ with Gaussian codebook is adopted. We define the coding rate $\mathrm{R}=k / n_{s}$, where $n_{s}$ is the number of symbols within each packet after channel encoding

\footnotetext{
${ }^{2}$ The channel load corresponds to the logical load G, since it takes into consideration the net information transmitted, depurated from the number of replicas per user $d$.

${ }^{3}$ It is important to underline that, the concept of VF has been firstly introduced in ACRDA [28], and was not present neither in CRA nor in the first statement of ECRA [31].
} 
and modulation. We denote with $T_{s}$ the duration of a symbol so that $T_{p}=T_{s} n_{s}$. Replicas are then transmitted through an additive white Gaussian noise (AWGN) channel.

Let us consider the transmitted signal $x^{(u)}$ of the $u$-th user,

$$
x^{(u)}(t)=\sum_{i=0}^{n_{s}-1} a_{i}^{(u)} g\left(t-i T_{s}\right) .
$$

Where $\boldsymbol{a}^{(u)}=\left(a_{0}^{(u)}, a_{1}^{(u)}, \ldots, a_{n_{s}-1}^{(u)}\right)$ is the codeword of user $u$ and $g(t)=\mathcal{F}^{-1}\{\sqrt{\operatorname{CR}(f)}\}$ is the pulse shape, being $\mathrm{CR}(f)$ the frequency response of the raised cosine filter. The generic user $u$ signal is affected by a frequency offset, modeled as an uniformly distributed random variable $f^{(u)} \sim \mathcal{U}\left[-f_{\max } ; f_{\max }\right]$, and a sampling epoch (cfn. [32] Chapter 2), also modeled as an uniformly distributed random variable $\epsilon^{(u)} \sim \mathcal{U}\left[0 ; T_{s}\right)$. Both frequency offset and sampling epoch are common to each replica of the same user, but independent user by user. The phase offset is modeled as a random variable uniformly distributed between 0 and $2 \pi$, i.e. $\varphi^{(u, r)} \sim \mathcal{U}[0 ; 2 \pi)$, and it is assumed to be independent replica by replica. Assuming that $f_{\max } T_{s} \ll 1$, the received signal $y(t)$, after matched filtering, can be approximated as

$$
\begin{aligned}
y(t) & \left.\cong \sum_{u} \sum_{r=0}^{\mathrm{d}-1} \tilde{x}^{(u)}\left(t-\epsilon^{(u)}-T^{(u, r)}-t_{0}^{(u)}\right) e^{j\left(2 \pi f^{(u)}+\varphi^{(u, r)}\right.}\right) \\
& +n(t)
\end{aligned}
$$

with $\tilde{x}^{(u)}=\sum_{i=0}^{n_{s}-1} a_{i}^{(u)} \tilde{g}\left(t-i T_{s}\right)$, where $\tilde{g}(t)=\mathcal{F}^{-1}\{\mathrm{CR}(f)\}$. In equation (2), $T^{(u, r)}$ is the delay w.r.t. the VF frame start for user $u$ and replica $r$, while $t_{0}^{(u)}$ is the $u$-th user delay w.r.t. the common reference time. The noise term $n(t)$ is given by $n(t) \triangleq \nu(t) * h(t)$, where $\nu(t)$ is a white Gaussian process with single-sided power spectral density $N_{0}$ and $h(t)$ is the matched filter (MF) impulse response of the root raised cosine filter, i.e. $h(t)=\mathcal{F}^{-1}\{\sqrt{\mathrm{CR}(f)}\}$.

For the $u$-th user, $r$-th replica, assuming an ideal estimate of the sampling epoch $\epsilon^{(u)}$, the frequency offset $f^{(u)}$ and the phase offset $\varphi^{(u, r)}$, the discrete-time version of the received signal $\boldsymbol{y}^{(u, r)}=\left(y_{0}^{(u, r)}, \ldots, y_{n_{s}-1}^{(u, r)}\right)$ is given by

$$
\boldsymbol{y}^{(u, r)}=\boldsymbol{a}^{(u)}+\boldsymbol{z}^{(u, r)}+\boldsymbol{n} .
$$

Here $\boldsymbol{z}^{(u, r)}$ is the interference contribution over the user$u$ replica- $r$ signal and $\boldsymbol{n}=\left(n_{0}, \ldots, n_{n_{s}-1}\right)$ are the samples of a complex discrete white Gaussian process with $n_{i} \sim \mathcal{C N}\left(0,2 \sigma_{n}^{2}\right)$.

The instantaneous signal-to-interference and noise ratio (SINR) $\gamma$ for the $i$-th sample of the $u$-th user $r$-th replica is

$$
\gamma_{i}^{(u, r)}=\frac{\mathrm{P}_{i}^{(u)}}{\mathrm{N}+\mathrm{Z}_{i}^{(u, r)}}
$$

with $\mathrm{P}_{i}^{(u)} \triangleq \mathbb{E}\left[\left|a_{i}^{(u)}\right|^{2}\right], \mathrm{N}=2 \sigma_{n}^{2}$ and $\mathrm{Z}_{i}^{(u, r)} \triangleq \mathbb{E}\left[\left|z_{i}^{(u, r)}\right|^{2}\right]$, which is the aggregate interference power contribution on the $i$-th sample of the considered replica. Throughout the paper, we assume that all users are received with the same power, i.e. perfect power control is adopted. Hence, $\mathrm{P}_{i}^{(u)}=\mathrm{P}$ and
$\mathrm{Z}_{i}^{(u, r)}=m_{i}^{(u, r)} \mathrm{P}$, where $m_{i}^{(u, r)}$ denotes the number of active interferers over the $i$-th symbol of the $u$-th user $r$-th replica. The aggregate interference is a discrete Gaussian process, with $z_{i} \sim \mathcal{C N}\left(0, m_{i}^{(u, r)} \mathrm{P}\right)$, and the SINR thus becomes

$$
\gamma_{i}^{(u, r)}=\frac{\mathrm{P}}{\mathrm{N}+m_{i}^{(u, r)} \mathrm{P}} .
$$

The SINR vector over the $n_{s}$ symbols of the considered replica is denoted with $\Gamma^{(u, r)}=\left(\gamma_{0}^{(u, r)}, \gamma_{1}^{(u, r)}, \ldots, \gamma_{n_{s}-1}^{(u, r)}\right)$.

\section{A. Modeling of the Decoding Process}

Typically, the destructive collision channel model is adopted [19] in the analysis of the medium access (MAC) layer of RA protocols. This physical layer abstraction assumes that, only packets received collision-free can be correctly decoded, while all packets involved in collisions are lost. This assumption is, in general, inaccurate when packets are protected with an error correcting code, and for asynchronous schemes specifically, is particularly pessimistic. ${ }^{4}$ In fact, interference can be counteracted by the error correction code, and some collisions can be resolved.

Motivated by this, we resort to a block interference model [33] given by $n_{s}$ subsequent Gaussian channels [34] (one for each replica symbol), where the $i$-th channel is characterized by a signal-to-noise ratio (SNR) $\gamma_{i}{ }^{5}$ Similarly to [35], the idea is to take into account the mutual information carried by each replica symbol, and then compute the average over the entire replica. Leveraging on the Gaussian assumption of both the signals and noise, ${ }^{6}$ the instantaneous mutual information over the $i$-th channel $\mathrm{I}\left(\gamma_{i}\right)$ is

$$
\mathrm{I}\left(\gamma_{i}\right)=\log _{2}\left(1+\gamma_{i}\right) .
$$

Differently from the classical parallel Gaussian channel problem of finding the best power allocation per channel, in order to maximize capacity (cf. Chapter 10.4 of [34]), here channel state information (CSI) is not present at the transmitter, since the interference contribution cannot be predicted due to the uncoordinated user transmissions. Therefore, the power allocation over the channels, i.e. symbols of the replica, is kept constant and is not subject to optimization. The instantaneous mutual information, averaged over the $n_{s}$ channels, is

$$
\mathrm{I}(\Gamma)=\frac{1}{n_{s}} \sum_{i=0}^{n_{s}-1} \mathrm{I}\left(\gamma_{i}\right)=\frac{1}{n_{s}} \sum_{i=0}^{n_{s}-1} \log _{2}\left(1+\gamma_{i}\right) .
$$

The interference has been modeled similarly in [35]. We introduce a binary variable $\mathcal{D}$, modelling the decoding process, such that

$$
\begin{array}{ll}
\mathcal{D}=1 & \text { if decoding succeeds } \\
\mathcal{D}=0 & \text { otherwise. }
\end{array}
$$

${ }^{4}$ Also for slot synchronous RA with powerful error correcting codes, decoding of packets may be possible even in presence of interference.

${ }^{5} \mathrm{We}$ are omitting here the superscript $(u, r)$ for ease of notation.

${ }^{6} \mathrm{We}$ shall point out that, under some specific conditions, the Gaussian assumption for the interference can also be a good approximation for linear modulated and Turbo encoded signals. See e.g. [14]. 
We have

$$
\mathcal{D}=\mathbb{I}\{\mathrm{R} \leq \mathrm{I}(\Gamma)\}
$$

where $\mathbb{I}\{X\}$ denotes the indicator (Inverson) function. ${ }^{7}$ Observe that, the destructive collision model is a special case, where the rate $\mathrm{R}$ is chosen such that only packets collisionfree can be succesfully decoded, i.e. $R=\log _{2}\left(1+\frac{\mathrm{P}}{\mathrm{N}}\right)$. The decoding process model based on the threshold induced by the selected rate, has some non-negligible effect on the performance with respect to more accurate models, that take into account the specific channel code and block length. Nevertheless, it is a good first approximation for highlighting the improvements given by the proposed scheme.

\section{B. Enhanced Contention Resolution ALOHA Decoding Algo- rithm}

At the receiver, ECRA follows a two phase procedure, in order to decode the received packets. The receiver will operate with a sliding window, similarly to [28], [38]. The decoder starts operating on the first $W$ samples, with $W$ the designed window size.

1) SIC phase: During the first phase, the decoder seeks for replicas that can be successfully decoded. Making the use of the example shown in Fig. 1 where a degree $d=2$ has been selected, we describe the SIC procedure. The first replica that can be decoded is $C_{2}$, assuming that very limited interference can be counteracted by the error correcting code. Thanks to the pointer to the position of all replicas of this user in the header, the decoder can retrieve the position of replica $C_{1}$ as well. In this way, replica $C_{2}$ can be re-encoded, re-modulated, frequency offset and sampling epoch are superimposed on the signal, and its interference contribution is removed from both locations within the received signal. In the following we assume ideal SIC, i.e. the entire interference contribution is removed from the received signal. Replica $A_{2}$ is now released from the interference, and can also be correctly decoded. In this scenario, the SIC procedure is iterated until none of the replicas can be successfully decoded anymore. At the end of SIC, users $1,2,3,4$ can be correctly decoded, while users 5 and 6 remain still unresolved, due to the presence of reciprocal interference that cannot be counteracted by the channel code. ${ }^{8}$

2) Combining phase: In the second phase of ECRA, combining techniques are applied on the received packets unable to be decoded in first phase, and on these combined observations decoding is attempted. The formal definition of a combined observation is as follows:

Definition 1 (Combined observation). Consider the $\mathrm{d}$ observations of the $u$-th packet, $\boldsymbol{y}^{(u, 1)}, \boldsymbol{y}^{(u, 2)}, \ldots, \boldsymbol{y}^{(u, \mathrm{~d})}$ with $\boldsymbol{y}^{(u, r)}=\left(y_{0}^{(u, r)}, y_{1}^{(u, r)}, \ldots, y_{n_{s}-1}^{(u, r)}\right)$. We define the combined

\footnotetext{
${ }^{7}$ This model allows to take into account features like channel coding, multipacket reception and capture effect [36], [37].

${ }^{8}$ In this example, we assumed a channel code able to counteract only very limited interference. In general, the code rate can be lowered to support higher levels of interference, thus possibly, leading to successful decoding. Unfortunately, there are still interference patterns in which the channel code alone is not able to counteract the interference, and prevents SIC to resolve the collision.
}

observation the vector

$$
\boldsymbol{y}^{(u)}=\left(y_{0}^{(u)}, y_{1}^{(u)}, \ldots, y_{n_{s}-1}^{(u)}\right)
$$

with $y_{i}^{(u)}$ being a suitable function of the individual observation samples $y_{i}^{(u, 1)}, y_{i}^{(u, 2)}, \ldots, y_{i}^{(u, \mathrm{~d})}$, i.e.

$$
y_{i}^{(u)}:=f\left(y_{i}^{(u, 1)}, y_{i}^{(u, 2)}, \ldots, y_{i}^{(u, \mathrm{~d})}\right) .
$$

Any of selection combining (SC), equal-gain combining (EGC) or maximal-ratio combining (MRC) [29], [30] can be applied in the second phase of ECRA, although our focus will be on SC and MRC. If SC is adopted, the combined observation is composed by the replica sections with the highest SINR, i.e. for each observed symbol, the selection combiner chooses the replica with the highest SINR. Hence, the instantaneous mutual information of the $u$-th user combined observation, $i$-th symbol after $\mathrm{SC}$ is

$$
\mathrm{I}\left(\gamma_{i}^{\mathrm{S}}\right)=\log _{2}\left(1+\max _{r}\left[\gamma_{i}^{(u, r)}\right]\right)
$$

Fig. 2 depicts the situation at the beginning of the second phase, for the example presented in Fig. 1. The selection combiner selects the first part of replica $F_{1}$, and the second part of $F_{2}$, building a combined observation free from interference.

In ECRA maximal-ratio combining (ECRA-MRC) instead, each replica observed symbol of a given user is weighted proportional to its root mean squared received signal level [29]. In this way, the SINR at the output of the combiner is the sum of all replicas SINRs. It is also known from literature that, MRC is optimal if the interference on each replica is independent [39]. The instantaneous mutual information of the $u$-th user combined observation $i$-th symbol after MRC is

$$
\mathrm{I}\left(\gamma_{i}^{\mathrm{M}}\right)=\log _{2}\left(1+\sum_{r=1}^{\mathrm{d}} \gamma_{i}^{(u, r)}\right)
$$

The decoder outcome after SC or MRC is modeled substituting the expression of $\mathrm{I}\left(\gamma_{i}\right)$ with I $\left(\gamma_{i}^{\mathrm{S}}\right)$ or I $\left(\gamma_{i}^{\mathrm{M}}\right)$ in equation (7), and adopting the same condition as in equation (9). When decoding is successful, the packet is re-encoded, re-modulated and its interference contribution is removed in all the positions within the frame, where the replicas of the decoded user are placed. Combining and SIC are iterated until, either all users are correctly decoded, or no more packets are present in the receiver window $W$. The receiver window is then shifted forward by $\Delta W$ samples, and the procedure starts again.

\section{Summary and Comments}

The second step of ECRA needs complete knowledge of the replicas position of the remaining users in the frame. Although stringent, this requirement can be addressed in two practical ways: either adopting dedicated pointers to the replicas locations in the header, or exploiting correlation techniques for detection and combining of the replicas, prior to decoding. The former solution adopts a pseudo-random seed, that is used at the receiver, for retrieving the information on all replicas position of the decoded user. This option was proposed first in [11], for slotted protocols, but can be extended also to ECRA. 


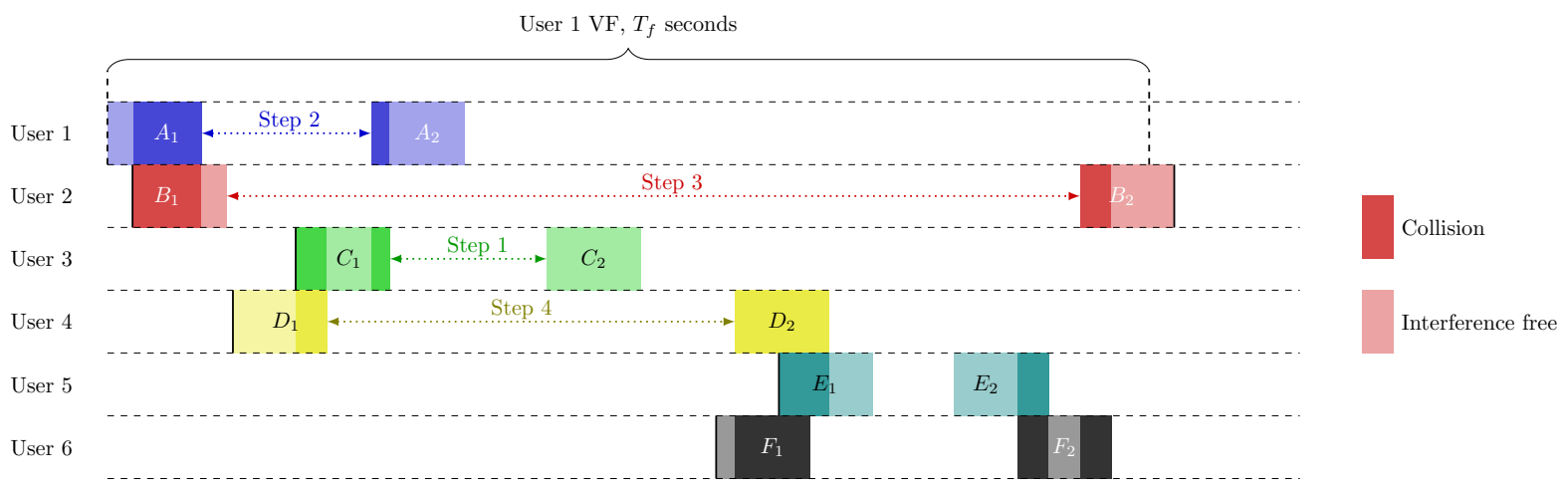

Fig. 1: SIC procedure in ECRA, first phase. The decoder starts looking for replicas that can be successfully decoded. The first to be found is replica $C_{2}$, which is collision-free. After successfully decoding, the information on the location of replica $C_{1}$ is retrieved from the header. So, the data carried by $C_{2}$ can be re-encoded, re-modulated, frequency offset and sampling epoch are superimposed on the signal, and its interference contribution is removed from both locations within the received signal. The interference caused on replica $A_{2}$ is now removed. The decoder can successfully decode also replica $A_{2}$, and applying the same procedure, remove its interference together with the one of replica $A_{1}$. Now, replica $B_{1}$ is collision-free, can be successfully decoded and its interference contribution together with the one of replica $B_{2}$ can be removed. Finally, replica $D_{1}$ also collision-free is correctly decoded, and removed from the received signal together with its twin $D_{2}$. Unfortunately user 5 and 6 replicas are in a collision pattern that cannot be resolved by SIC only, and still remain in the received signal after the end of the first phase.

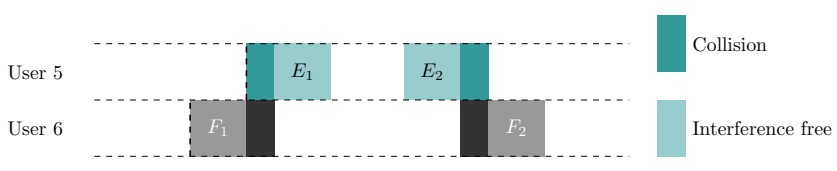

Fig. 2: Example of a collision pattern blocking SIC. Different portions of replicas $F_{1}$ and $F_{2}$ are collision-free. When SC is applied, ECRA selects these portions, creates a combined observation and attempts decoding on it.

The pseudo-random seed is used for generating the relative time offset between replicas, and together with the replica sequence number, allows to identify the replicas locations. In [40] it is shown that, in the low to moderate channel traffic regions, low probability of interference in the header can be found. In the high channel traffic region instead, replicating the header twice is beneficial. Moreover, if a dedicated channel code is introduced for protecting the header, lower header loss probability are expected. ${ }^{9}$

When correlation techniques are adopted, no overhead due to a dedicated field in the header is necessary, and replicas are detected and combined before decoding [41]. A two-phase procedure is proposed in [41]: first, transmitted replicas are detected, and second, replicas belonging to the same user are matched. Specifically, exploiting the presence of preamble at the start of a replica, common to every user and replica, a non-coherent soft-correlation metric is adopted for detection. The correlator needs to distinguish between two hypothesis, the presence of a preamble, or simply the presence of noise and, potentially, interference. A threshold test is exploited, and the correlator outputs a decision for each sample of the recorded signal. Whenever the correlator exceeds a defined threshold, the sample is declared as start of a candidate replica. In the second phase, for each candidate replica, the one belonging to the same user are sought, within the set of

\footnotetext{
${ }^{9}$ Dedicated channel code applied to the headers, can allow retrieving the information about replica locations, although the packet itself is cannot be decoded due to collisions.
}

candidate replicas. Similarly to the first phase, a non-coherent soft-correlation metric is a adopted. On the other hand, in the second phase, we can exploit the information along the whole packet, since replicas of the same user are identical. This helps the performance to improve enormously. In fact, we can use correlation over thousands of symbols, instead of only few tens as per the preamble. In the mentioned work, we proposed also two enhancements to the technique. In the first place, if users transmit their replicas within discrete intervals, the second phase can benefit of a reduction in complexity. Candidate replicas sent out of the discrete intervals can be discarded a-priori, without requiring the correlation to take place. Furthermore, if the interference power is estimated, the first phase can be enhanced. Starting from the approximate likelihood ratio test, we developed an interference-aware metric. In the paper, results on both first and second phase are presented, showing very good performance. The comparison between ideal detection and matching compared to the twophase approach shows a very limited degradation in spectral efficiency.

A similar correlation approach have been proposed by the authors of [20], but in a time-synchronous scenario. Two main differences can be identified, firstly the technique proposed for the asynchronous scenario adopts a two-phase non-coherent correlation approach where, in the first phase only, the detection is performed. Secondly, a discretization of the time instants in which a user can transmit its replicas is proposed, so to reduce the number of correlations to be performed. In other words, not all combinations of candidate replicas are allowed.

MRC combining technique requires the knowledge of the SINR symbol-by-symbol, in order to choose the optimal weights [29] beforehand the combination is done. In case this information cannot be retrieved, combining can be applied with equal weights for all the symbols, i.e. EGC.

The scenarios under consideration in the work of [42], and its extension [43], are similar to the one that can block the 
SIC procedure (see Fig. 2), although some differences in the solutions between their work and ECRA can be identified. ECRA creates the combined observation and tries decoding on it, while [42] requires an iterative demodulation procedure within packet portions, that may increases the overall packet decoding delay. Furthermore, in [42] an error in one decoded bit propagates to the entire packet unless compensated by further errors. This is due to the iterative procedure applied, which subtracts the uncorrect bit from the same packet in the second collision, while in ECRA an error in one decoded bit will not affect any other portion of the packet.

\section{Packet Loss Rate Analysis at Low Channel LOAD}

In this Section a PLR approximation, tight for low channel load conditions, is derived. Packet losses are caused by particular interference patterns that SIC is not able to resolve. In the slot synchronous RA protocols, these patterns are analogous to the stopping sets present in low density parity check (LDPC) codes [44], and can be analyzed exploiting tools from coding theory, and graph theory. In the asynchronous RA schemes, a graph representation is not straightforward, since no discrete objects as slots are present anymore. Therefore, we resort to investigate the collision patterns that involve two users only, with a generic degree $d$, and conjecture that these are the patterns driving the PLR, especially at low channel loads. In the next section, the approximation of the PLR is compared with Monte Carlo simulations, in order to verify its tightness. A set of definitions are required for the analysis.

Definition 2 (Collision cluster $\mathcal{S}$ ). Consider a subset $\mathcal{S}$ of users. Assume that packets of all users in $\mathcal{S}^{c}$ (complementary of the subset $\mathcal{S}$ ) have been successfully decoded. The subset $\mathcal{S}$ is referred to as collision cluster iff no packet replicas for the users in $\mathcal{S}$ is collision-free.

Under the assumption of collision channel, none of the users in the collision cluster can be successfully decoded. Conversely, when a channel code $\mathcal{C}$ is employed by each transmitted packet, the collision cluster might be resolvable, leading to the following definition.

Definition 3 ( $\mathcal{C}$-unresolvable collision pattern). Given each packet encoded with a channel code $\mathcal{C}$, a $\mathcal{C}$-unresolvable collision pattern $(\mathcal{C}$-UCP) $\mathcal{L}$ is a collision cluster where no user in the set can be successfully decoded.

Every $\mathcal{C}$-UCP is also a collision cluster, but not viceversa. In order to evaluate the probability of $\mathcal{C}$-UCP involving two users only, a generalization of the definition of vulnerable period [13] is required.

Definition $4(\mathcal{C}$-vulnerable period for $|\mathcal{S}|=2)$. Consider the transmission of a packet protected with a channel code $\mathcal{C}$ between time $\tau$ and $\tau+T_{p}$. The packet's $\mathcal{C}$-vulnerable period is the interval of time $\left[\tau-\tau_{l}^{*}, \tau+\tau_{r}^{*}\right]$ in which the presence of a single interferer leads to a failure in the decoding.

Hence, the vulnerable period duration $T_{v}$ is defined as

$$
T_{v}=\tau_{l}^{*}+\tau_{r}^{*} .
$$

In slotted synchronous schemes under the collision channel model, $\tau_{l}^{*}=0$ and $\tau_{r}^{*}=T_{p}$, so $T_{v}=T_{p}$. For asynchronous schemes in general and therefore for ECRA, it holds $\tau_{l}^{*}=\tau_{r}^{*} \triangleq \tau^{*}$. The vulnerable period duration for asynchronous schemes is $T_{v}=2 \tau^{*}$. Considering the collision channel model, the vulnerable period duration is then $T_{v}=$ $2 T_{p}$. So, the duration of packets' vulnerable period is doubled in asynchronous schemes w.r.t. comparable synchronous ones [13]. Examples of collision clusters, $\mathcal{C}$-unresolvable collision patterns and vulnerable periods are provided in Fig. 3.

\section{A. Packet Loss Rate Approximation}

In this section, we derive an approximation of the PLR, denoted as $\mathrm{p}_{l}$. The approach follows [45], extending the investigation to asynchronous schemes. Let us consider the user $u$. We denote with $\mathcal{L}_{\mathrm{S}}$ the set of all possible $\mathcal{C}$-UCP that cause the loss of user $u$ packets, with $\mathcal{L}$ the index of the $\mathcal{C}$ UCP set considered when writing the union bound, and with $\mathcal{L}^{*}$ the unique type of $\mathcal{C}$-UCP that we assume to drive the PLR performance $\mathrm{p}_{l}$. Let $n_{p}=T_{f} / T_{p}$ denote the VF length measured in packet durations, and $n_{v}=\left\lfloor T_{f} / T_{v}\right\rfloor$ denote the number of disjoint vulnerable periods per VF. ${ }^{10}$ Clearly $n_{p} \geq \mathrm{d}$. The PLR can be approximated with

$$
\begin{aligned}
\mathrm{p}_{l} & =\operatorname{Pr}\left\{\bigcup_{\mathcal{L} \in \mathcal{L}_{\mathrm{S}}} u \in \mathcal{L}\right\} \leq \sum_{\mathcal{L} \in \mathcal{L}_{\mathrm{S}}} \operatorname{Pr}\{u \in \mathcal{L}\} \approx \operatorname{Pr}\left\{u \in \mathcal{L}^{*}\right\} \\
& =\sum_{m=2}^{\infty} \frac{e^{-n_{p} \mathrm{G}}\left(n_{p} \mathrm{G}\right)^{m}}{m !} \operatorname{Pr}\left\{u \in \mathcal{L}^{*} \mid m\right\}
\end{aligned}
$$

The probability $\mathrm{p}_{l}$ is first bounded from above with the union bound and then approximated considering only one type of $\mathcal{C}$ $\mathrm{UCP}$, i.e. $\mathcal{L}^{*}$. Finally, we take the expectation of the number of active users over the $u$-th user VF. The $\mathcal{C}$-UCP $\mathcal{L}^{*}$ considered in the analysis is formed by two users only with a generic degree $\mathrm{d}$. The probability that, the considered user $u$ belongs to the $\mathcal{C}$-UCP $\mathcal{L}^{*}$ formed by two users, is approximated as

$$
\operatorname{Pr}\left\{u \in \mathcal{L}^{*} \mid m\right\} \approx \frac{\alpha_{u}\left(\mathcal{L}^{*}, m\right) \beta_{d}\left(\mathcal{L}^{*}\right)}{\beta_{u-d}\left(\mathcal{L}^{*}\right)} \frac{2}{m} .
$$

Where, we denote with $\alpha_{u}\left(\mathcal{L}^{*}, m\right)$ the number of possible combinations of $m$ users, taken two by two, i.e. $\alpha_{u}\left(\mathcal{L}^{*}, m\right)=\left(\begin{array}{c}m \\ 2\end{array}\right)$. The second term $\beta_{d}\left(\mathcal{L}^{*}\right)$, accounts for the number possible placements of $d$ replicas. Since the first replica of user $u$ is sent immediately, only the remaining $\mathrm{d}-1$ are free to be sent within a delay selected uniformly at random, and not exceeding a VF duration. In terms of vulnerable periods, the number of possible placements of the remaining $\mathrm{d}-1$ replicas is $\beta_{d}\left(\mathcal{L}^{*}\right) \approx\left(\begin{array}{c}n_{v}-1 \\ \mathrm{~d}-1\end{array}\right)$. Similarly, the number of ways in which two users can select their position for the replicas, follows $\beta_{u-d}\left(\mathcal{L}^{*}\right) \approx n_{v}\left(\begin{array}{c}n_{v}-1 \\ \mathrm{~d}-1\end{array}\right)^{2}$. The probability that the two users (including user $u$ ) placed all their $\mathrm{d}$ replicas in the reciprocal vulnerable periods is $1 / \beta_{u-d}\left(\mathcal{L}^{*}\right)$. Finally,

\footnotetext{
${ }^{10}$ It is possible that, the vulnerable period of the replicas of one user overlap. This is the case when the relative delay between replicas is smaller than the vulnerable period. On the other hand, this probability is reasonably small for virtual frames of interest.
} 

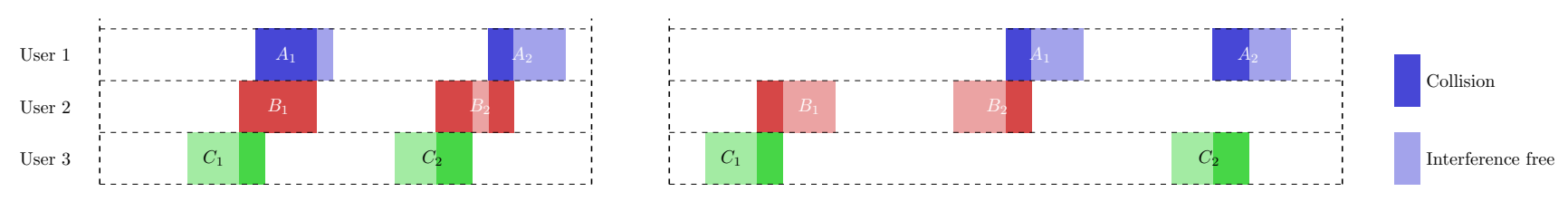

(a) Two types of collision clusters with $|\mathcal{S}|=3$ and with $\mathrm{d}=2$. If the error correcting code $\mathcal{C}$ is not able to counteract the interference of any replica, the collision clusters are also $\mathcal{C}$-unresolvable collision patterns.

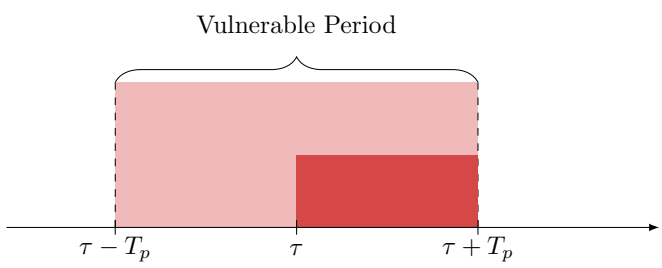

(b) Vulnerable period for a packet transmitted with an asynchronous RA protocol, under the collision channel model.

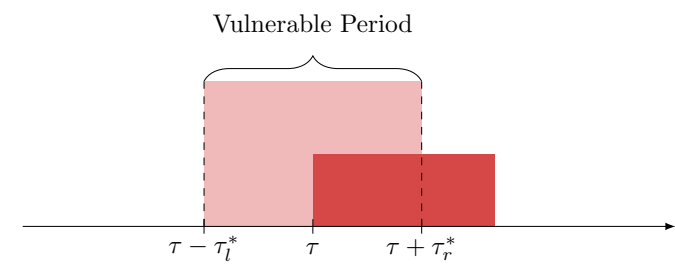

(c) Vulnerable period for a packet transmitted with an asynchronous RA protocol, with an error correcting code $\mathcal{C}$.

Fig. 3: Examples of collision clusters, $\mathcal{C}$-unresolvable collision patterns and vulnerable periods.

the probability that the two users belong to the $\mathcal{C}$-UCP $\mathcal{L}^{*}$ is $2 / m$. Substituting into equation (16) we get

$$
\operatorname{Pr}\left\{u \in \mathcal{L}^{*} \mid m\right\} \approx \frac{\left(\begin{array}{c}
m \\
2
\end{array}\right)}{n_{v}\left(\begin{array}{c}
n_{v}-1 \\
\mathrm{~d}-1
\end{array}\right)} \frac{2}{m}=\frac{\left(\begin{array}{c}
m \\
2
\end{array}\right)}{\mathrm{d}\left(\begin{array}{c}
n_{v} \\
\mathrm{~d}
\end{array}\right)} \frac{2}{m} .
$$

Finally, inserting in equation (15) the result of equation (17) we can approximate the PLR $\mathrm{p}_{l}$ as

$$
\mathrm{p}_{l} \approx \sum_{m=2}^{\infty} \frac{e^{-n_{p} \mathrm{G}}\left(n_{p} \mathrm{G}\right)^{m}}{m !} \frac{\left(\begin{array}{c}
m \\
2
\end{array}\right)}{\mathrm{d}\left(\begin{array}{c}
n_{v} \\
\mathrm{~d}
\end{array}\right)} \frac{2}{m} .
$$

The PLR approximation directly depends on the vulnerable period duration, via $n_{v}$. In the next Sections, the vulnerable period duration is computed for two scenarios of interest, including the MRC case.

\section{B. Vulnerable Period Duration for Asynchronous RA with forward error correction (FEC)}

In this scenario, packets, or replicas for schemes adopting them, are protected by a channel code so that not all collisions are destructive. The only $\mathcal{C}$-UCP to be considered is the one involving two users and their packets or replicas. We recall that perfect power control is assumed so that both users are received with the same power $P$. Without loss of generality, we focus on a specific packet, or replica, involved in an $\mathcal{C}$ UCP of type $\mathcal{L}^{*}$, which has a first section free of interference and a second part interfered. The selected rate $\mathrm{R}$ determines what it is the minimum fraction of interference-free packet, or replica, $\varphi_{a}$ that still allows correct decoding, i.e.

$$
\varphi_{a} \log _{2}\left(1+\frac{\mathrm{P}}{\mathrm{N}}\right)+\left(1-\varphi_{a}\right) \log _{2}\left(1+\frac{\mathrm{P}}{\mathrm{N}+\mathrm{P}}\right)=\mathrm{R} \text {. }
$$

For the sake of simplicity we denote with

$$
\begin{aligned}
& \mathrm{R}_{f}=\log _{2}\left(1+\frac{\mathrm{P}}{\mathrm{N}}\right) \\
& \mathrm{R}_{i}=\log _{2}\left(1+\frac{\mathrm{P}}{\mathrm{N}+\mathrm{P}}\right)
\end{aligned}
$$

and we solve equation (19) for $\varphi_{a}$

$$
\varphi_{a}=\frac{\mathrm{R}-\mathrm{R}_{i}}{\mathrm{R}_{f}-\mathrm{R}_{i}} .
$$

Equation (21) is valid for $\mathrm{R} \geq \log _{2}\left(1+\frac{\mathrm{P}}{\mathrm{N}+\mathrm{P}}\right)$. In fact, for $\mathrm{R}<\mathrm{R}_{i}$, no $\mathcal{C}$-UCPs involving only two users can be observed, and regardless the level of interference, packets involved in collisions with only one other packet can be always decoded. In this way,

$$
\varphi_{a}=\left\{\begin{aligned}
\frac{\mathrm{R}-\mathrm{R}_{i}}{\mathrm{R}_{f}-\mathrm{R}_{i}} & \text { for } \mathrm{R} \geq \mathrm{R}_{i} \\
0 & \text { for } \mathrm{R}<\mathrm{R}_{i}
\end{aligned}\right.
$$

It is worth noticing that $\varphi_{a}$ is constrained to $0 \leq \varphi_{a} \leq 1$, since the selectable rate $R$ is $R \leq \log _{2}\left(1+\frac{P}{N}\right)=R_{f}$ for reliable communication.

In this way, $\tau^{*}=\varphi_{a} T_{p}$ and therefore the vulnerable period is reduced to $T_{v}=2 \tau^{*}=2 \varphi_{a} T_{p}$. And finally $n_{v}=\left\lfloor T_{f} / T_{v}\right\rfloor=\left\lfloor T_{f} /\left(2 \varphi_{a} T_{p}\right)\right\rfloor$. Inserting the value of $n_{v}$ in equation (18) gives the final expression of the PLR approximation for asynchronous RA schemes using replicas. Note that for $\varphi_{a} \rightarrow 0, n_{v} \rightarrow+\infty$ and therefore the PLR approximation in equation (18) tends to 0 .

\section{Vulnerable Period Duration for Asynchronous RA with $M R C$ and $\mathrm{d}=2$}

Similarly to the previous section, $\mathcal{L}^{*}$ is the considered $\mathcal{C}$ UCP where two users are interfering each other and they are received with the same power $P$. In this scenario the degree is fixed to $d=2$. Focus is on the combined observation, after MRC. Without loss of generality, it is assumed that the first section of both replicas is free of interference, while there is a second part where just one replica is interfered and finally there is the last part where both replicas are interfered. We aim at computing the minimum combined observation portion 
interference free $\varphi_{m}$, that is required for correctly decoding the user after MRC. It holds

$$
\begin{aligned}
& \varphi_{m} \log _{2}\left(1+2 \frac{\mathrm{P}}{\mathrm{N}}\right)+\mu \log _{2}\left(1+\frac{\mathrm{P}}{\mathrm{N}}+\frac{\mathrm{P}}{\mathrm{N}+\mathrm{P}}\right) \\
& +\left(1-\varphi_{m}-\mu\right) \log _{2}\left(1+2 \frac{\mathrm{P}}{\mathrm{N}+\mathrm{P}}\right)=\mathrm{R} .
\end{aligned}
$$

For the sake of simplicity, we denote with

$$
\begin{aligned}
\mathrm{R}_{f} & =\log _{2}\left(1+2 \frac{\mathrm{P}}{\mathrm{N}}\right) \\
\mathrm{R}_{i 1} & =\log _{2}\left(1+\frac{\mathrm{P}}{\mathrm{N}}+\frac{\mathrm{P}}{\mathrm{N}+\mathrm{P}}\right) \\
\mathrm{R}_{i 2} & =\log _{2}\left(1+2 \frac{\mathrm{P}}{\mathrm{N}+\mathrm{P}}\right) .
\end{aligned}
$$

So that equation (23) becomes

$$
\varphi_{m} \mathrm{R}_{f}+\mu \mathrm{R}_{i 1}+\left(1-\varphi_{m}-\mu\right) \mathrm{R}_{i 2}=\mathrm{R} .
$$

In order to solve equation (25), $\mu$ is expressed as a function of $\varphi_{m}$, as $\mu=\alpha \varphi_{m}$, where $0 \leq \alpha \leq\left(1-\varphi_{m}\right) / \varphi_{m}$. When $\alpha=0$, there are no portions where only one out of the two replicas is interfered, while $\alpha=\left(1-\varphi_{m}\right) / \varphi_{m}$ represents the case when there are no portions where both replicas are interfered. Resolving (25) for $\varphi_{m}$ gives

$$
\varphi_{m}=\frac{\mathrm{R}-\mathrm{R}_{i 2}}{\mathrm{R}_{f}-\mathrm{R}_{i 2}+\alpha\left(\mathrm{R}_{i 1}-\mathrm{R}_{i 2}\right)} .
$$

Also in this case, for $\mathrm{R}<\mathrm{R}_{i 2}, \varphi_{m}=0$ which means that no $\mathcal{C}$-UCP involving two replicas can be found,

$$
\varphi_{m}=\left\{\begin{aligned}
\frac{\mathrm{R}-\mathrm{R}_{i 2}}{\mathrm{R}_{f}-\mathrm{R}_{i 2}+\alpha\left(\mathrm{R}_{i 1}-\mathrm{R}_{i 2}\right)} & \text { for } \mathrm{R} \geq \mathrm{R}_{i 2} \\
0 & \text { for } \mathrm{R}<\mathrm{R}_{i 2}
\end{aligned}\right.
$$

The average vulnerable period duration over the two replicas is $T_{v}=2 \tau^{*}=2\left(\varphi_{m}+\frac{\mu}{2}\right) T_{p}=2 \varphi_{m}\left(1+\frac{\alpha}{2}\right) T_{p} \cdot{ }^{11}$ And finally $n_{v}=\left\lfloor T_{f} / T_{v}\right\rfloor=\left\lfloor T_{f} /\left(2 \varphi_{m}\left(1+\frac{\alpha}{2}\right) T_{p}\right)\right\rfloor$.

The presented analysis can be extended also to a higher number of replicas, i.e. $d>2$. The main difference will be in the number of packet sections that shall be taken into account, which corresponds to $d+1$, in general. The other key difference is in the number of parameters, which grows as $d$.

\section{Performance Analysis}

In this section, ECRA-SC and ECRA-MRC are compared with the reference CRA protocol, as well as with ALOHA. For this first comparison two metrics are considered, the PLR and the throughput. The throughput $\mathrm{S}$ is defined as the expected number of successfully decoded packets per packet duration $T_{p}$,

$$
\mathrm{S}=\left(1-\mathrm{p}_{l}\right) \mathrm{G} .
$$

The ECRA algorithm is also compared against slot synchronous RA protocols, as CRDSA. Since a channel code $\mathcal{C}$ is adopted in the proposed scheme, the throughput is not

\footnotetext{
${ }^{11}$ It is important to underline that, the expression of the average vulnerable period duration presented is valid no matter how the two replicas are interfered, i.e. also when the portions interfered are not both at the beginning of the packets.
}

anymore a sufficient metric. In fact, thanks to the use of error correcting code at physical layer, even with equal received power, a certain level of interference can be sustained and collisions may be resolved. The level of interference allowing correct decoding depends on the selected rate R. Lowering the rate enables to resolve a higher number of collisions, but reduces the information carried by each packet. This tradeoff is captured by the spectral efficiency $\xi$,

$$
\xi=\left(1-\mathrm{p}_{l}\right) \mathrm{GR} \quad[\text { bits/symbol]. }
$$

Although ECRA can outperform considerably the ALOHA protocol, it entails a larger transmit energy per packet. In fact, this scheme assumes to replicate each packet sent in the frame $d$ times. In order to take into account the increase in energy per packet, we follow the approach of [5], that was extended for slotted synchronous protocols as CRDSA and IRSA in [16]. The normalized capacity $\eta$ is defined as the ratio between the maximum achievable spectral efficiency of one of the examined RA scheme and the channel capacity of multiple access Gaussian channel under the same average power constraint. The average power constraint takes into account, the channel load and the number of replicas, so to directly reflect variations in the energy per packet. The idea is to compute the maximum spectral efficiency of the asynchronous MAC schemes (ECRA-SC or ECRA-MRC) and normalize it to the sum rate capacity of the multiple access Gaussian channel $\mathrm{C}_{g}=\log _{2}\left(1+\mathrm{P}_{g} / \mathrm{N}\right)$. This is done fixing the average aggregate received signal power $\mathrm{P}_{g}$ equal in all the schemes. In this way, for the RA protocols the user transmission power $\mathrm{P}_{t}$ takes into account the fact that the channel is used intermittently but $d$ times w.r.t. ALOHA, i.e. $\mathrm{P}_{t}=\frac{\mathrm{P}_{g}}{\mathrm{G} \cdot \mathrm{d}}$. The ultimate performance of the asynchronous RA schemes is given by the maximum spectral efficiency $\xi^{*}$ defined as

$$
\xi^{*}=\max _{R \in\left[0, . ., R^{*}\right]} S(G) R
$$

where for each channel traffic value, the rate $\mathrm{R}$ which maximizes the spectral efficiency is chosen. ${ }^{12}$ Unfortunately, the throughput expression $S(G)$ is not available in closed form for ECRA-SC and ECRA-MRC, so only a numerical evaluation of equation (30) is possible. The normalized capacity $\eta$ is defined as

$$
\eta=\frac{\xi^{*}}{\mathrm{C}_{g}}
$$

where, depending on the RA, a different expression of $\xi^{*}$ will be used.

\section{A. Numerical Results}

In the following, numerical results for ECRA-SC and ECRA-MRC schemes are presented. The packets sent by the users are composed by $k=1000$ bits, which translate into $n_{s}=(k / \mathbf{R})$ symbols. The transmission period is then $T_{p}=T_{s} n_{s}$. The VF duration $T_{f}$ is selected to be equal to 200

\footnotetext{
${ }^{12}$ The maximum rate for reliable communication $\mathrm{R}^{*}$ is $\mathrm{R}^{*}=\log _{2}\left(1+\mathrm{P}_{t} / \mathrm{N}\right)$ and depends upon the selected channel load G.
} 


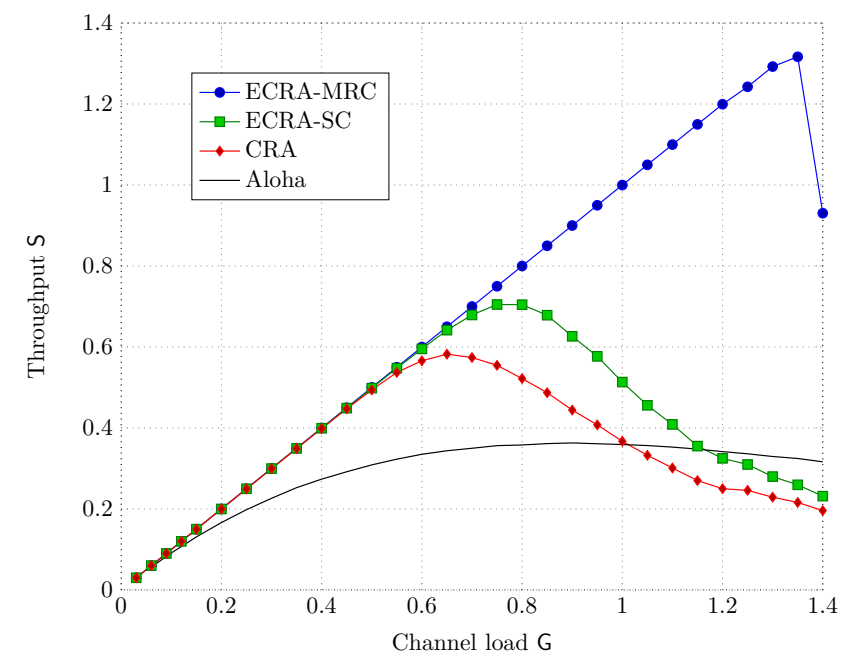

Fig. 4: Throughput $S$ vs. channel load $\mathrm{G}$ for ALOHA, CRA, ECRA-SC and ECRA-MRC, $\mathrm{P} / \mathrm{N}=6 \mathrm{~dB}$ and $\mathrm{R}=1.5$.

packet durations, i.e. $T_{f}=200 T_{p}$. We recall that, the number of users generating traffic follows a Poisson distribution, with mean $\mathrm{G}$ measured in packets per $T_{p}$ durations, and each of the users transmits $d=2$ replicas per generated packet. The decoder operates on a window of $W=3 T_{f}=600 T_{p}$ and once either the maximum number of SIC iterations is expired or no more packets can be successfully decoded, it is shifted forward by $\Delta W=20 T_{p}$. Ideal interference cancellation is assumed and the block interference model introduced in Section II-A is used for determining the successful decoding of a packet. Since the physical layer is abstracted, no frequency offset or phase offset are considered in the presented simulation results. $^{13}$

We present first the simulations of the throughput and PLR for both ECRA-SC and ECRA-MRC. For reference purposes also CRA and the ALOHA protocols are depicted in the figures. The assumptions are $\mathrm{P} / \mathrm{N}=6 \mathrm{~dB}$ and $\mathrm{R}=1.5$ equal for all users. In Fig. 4 the throughput $S$ vs. the channel load $\mathrm{G}$ is presented. ECRA-MRC largely outperforms both ECRA$\mathrm{SC}$ and CRA, reaching a maximum throughput of $\mathrm{S}=1.32$ at $\mathrm{G}=1.35$, which is more than twice the one of CRA, $\mathrm{S}=0.58$ and $89 \%$ of increase with respect to the one of ECRA$\mathrm{SC}, \mathrm{S}=0.70$. Furthermore, ECRA-MRC throughput follows linearly the channel load up to 1.3 packets per $T_{p}$, implying very small PLR. In fact, looking at the PLR performance in Fig. 5, ECRA-MRC is able to maintain the PLR below $10^{-3}$ for channel load below 1.2 packets per $T_{p}$. In other words for a target PLR of $\mathrm{p}_{l}=10^{-3}$, ECRA-MRC can be operated up to $\mathrm{G}=1.2$, while both ECRA-SC and CRA only up to $\mathrm{G} \cong 0.3$ and $\mathrm{G} \cong 0.2$ respectively. The gain of ECRA-MRC with respect to both ECRA-SC and CRA in terms of PLR is of at least one order of magnitude, except in the very high channel load region, where it largely exceeds this value. It is also shown in the figure, that this protocol is the only one that can maintain $\mathrm{p}_{l} \leq 10^{-4}$ for channel load values up to

\footnotetext{
${ }^{13}$ The impact of phase noise is not taken into account in the reported numerical results, due to the physical layer abstraction. The effect of phase noise is non-trivial and is therefore, left as part of future work.
}

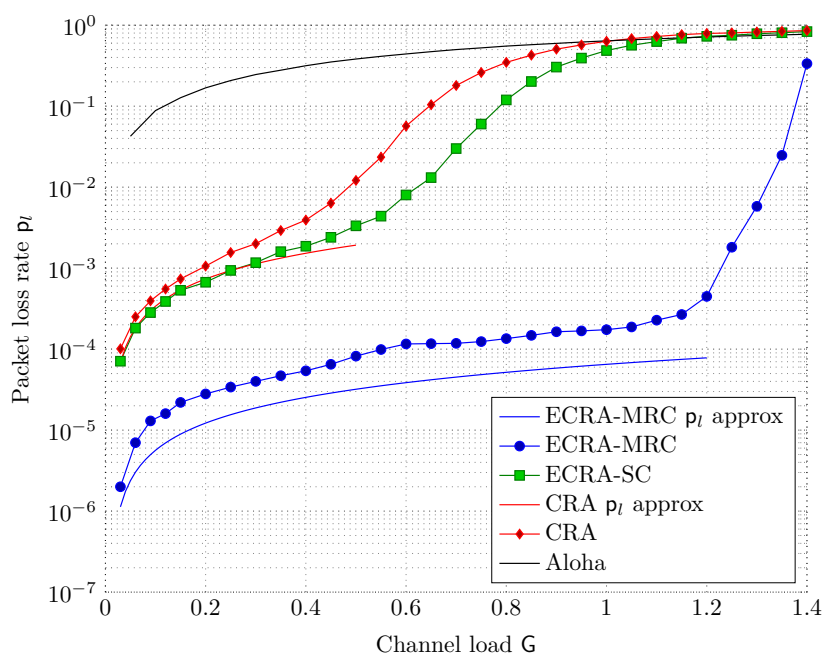

Fig. 5: Packet error rate $\mathrm{p}_{l}$ vs. channel load G for ALOHA, CRA, ECRA-SC and ECRA-MRC, $\mathrm{P} / \mathrm{N}=6 \mathrm{~dB}$ and $\mathrm{R}=1.5$.

$\mathrm{G}=0.6$. Very low PLR are particularly appealing in specific scenarios as satellite applications or control channels where reliability can be as important as efficiency.

In Fig. 5, the approximation on the $\mathrm{p}_{l}$ for both CRA and ECRA-MRC, derived in Section III-A, is also shown. This approximation takes into account only the errors coming from $\mathcal{C}$-UCPs involving two users, and for very limited channel load values is very close to the simulated $\mathrm{p}_{l}$. In particular, for the PLR approximation of CRA we used equation (22) and equation (18), while for the PLR approximation of ECRAMRC we used equations (27) and (18), with the numerically evaluated average $\alpha$. For CRA, when $\mathrm{G} \leq 0.3$, the approximation approaches the $\mathrm{p}_{l}$ simulated performance, while for increasing $\mathrm{G}$ the probability of having $\mathcal{C}$-UCPs involving more than two users starts to have an impact on $\mathrm{p}_{l}$ and therefore the approximation starts to become loose. Although a similar behavior can be found for the approximation of ECRA-MRC, interestingly the relative distance between the approximation and the simulations remains almost constant for a large range of channel load values.

In the second set of simulations, performance comparison of the slot synchronous scheme CRDSA with the asynchronous schemes CRA, ECRA-SC and ECRA-MRC is presented. The metrics used for the comparison are the spectral efficiency $\xi$ and the packet loss rate $\mathrm{p}_{l}$. We show numerical results for various rates. We select rate $\mathrm{R}=1.5$ for CRA and ECRA-SC, which is in line with the previous numerical results. Instead, for ECRA-MRC we choose two different rate values, i.e. $\mathrm{R}=1.5$ and $\mathrm{R}=0.67$, where the latter is adopted to present the benefits of strengthening the error correction capabilities. Finally, for CRDSA, we present results for $\mathrm{R}=2.31$, which corresponds to the best choice when decoding in presence of one interferer is not possible, and for $\mathrm{R}=0.67$, so to compare the performance at lower rate with ECRA-MRC. In Fig. 6, the spectral efficiency results are presented. Considering CRDSA with $\mathrm{R}=2.31$ and CRA, ECRA-SC with $\mathrm{R}=1.5$, we can observe that CRDSA can outperform considerably both CRA and ECRA-SC. Specifically, it shows a peak throughput 


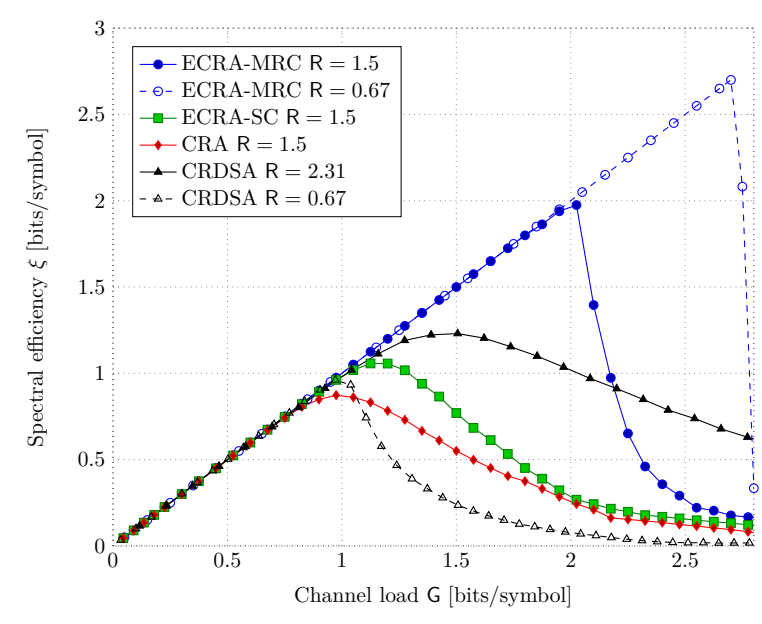

(a) Spectral efficiency at $\mathrm{P} / \mathrm{N}=6 \mathrm{~dB}$ for various rate values.

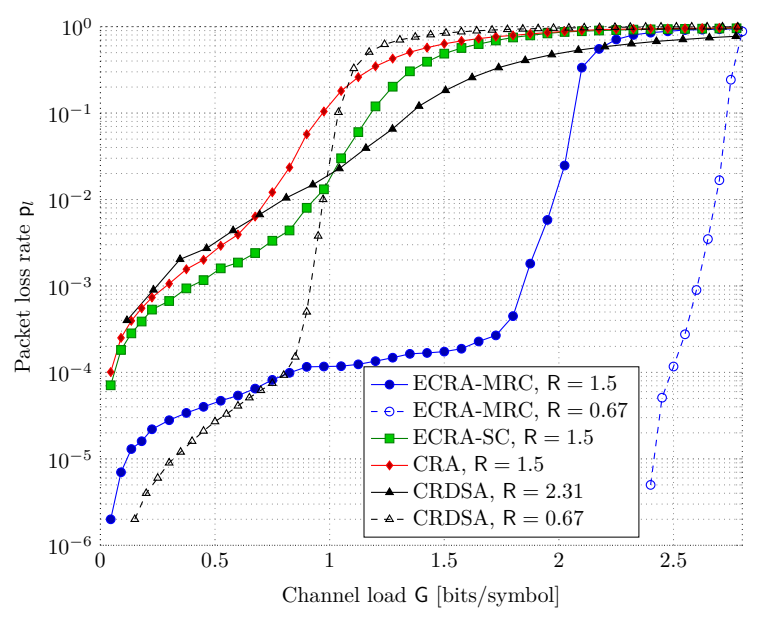

(b) Packet loss rate at $\mathrm{P} / \mathrm{N}=6 \mathrm{~dB}$ for various rate values.

Fig. 6: $\xi$ and PLR vs. channel load G for ECRA-SC, ECRA-MRC, CRA and CRDSA for different rate values.

that is $16 \%$ higher than ECRA-SC and $40 \%$ higher than CRA. Nonetheless, comparing the PLR results shown in Fig. $6 \mathrm{~b}$, we observe a very similar performance for low channel load values, up to $G=0.6[\mathrm{bits} / \mathrm{symbol}$. On the other hand, when this channel load is exceeded, CRDSA is able to gain on both CRA and ECRA-SC. The asynchronous ECRAMRC with $R=1.5$, instead, shows an outstanding gain of $60 \%$ in the maximum spectral efficiency of ECRA-MRC over CRDSA with $R=2.31$, reaching a spectral efficiency close to 2 [bits/symbol]. Reducing the rate to $\mathrm{R}=0.67$ leads to gains for both ECRA-MRC and CRDSA, looking at the PLR performance. For the former, PLR values below $10^{-6}$ are experienced for channel load up to $\mathrm{G}=2.4$ [bits/symbol], while for the latter it is achieved a PLR below $10^{-4}$ up to $\mathrm{G}=0.8$ [bits/symbol] and below $10^{-3}$ up to $\mathrm{G}=0.9$ [bits/symbol]. Comparing ECRA-MRC for $\mathrm{R}=1.5$ and for $\mathrm{R}=0.67$ we observe a drastic improvement in both the PLR performance as well as in the spectral efficiency when we select the lower rate. Although not presented in the figure due to space constraints, also for CRA and ECRA-SC a remarkable performance improvement can be shown for a rate of $R=0.67$ over $\mathrm{R}=1.5$.

The last set of simulations shows the comparison among ALOHA, CRA, ECRA-SC and ECRA-MRC, in terms of the normalized capacity $\eta . \mathrm{P}_{g} / \mathrm{N}=6 \mathrm{~dB}$ is selected and the results are presented in Fig. 7. The normalized capacity for ECRAMRC can reach up to $75 \%$ of the MAC channel capacity, for a channel load $\mathrm{G}=5$ with rate $\mathrm{R} \cong 0.35$; see Fig. $7 \mathrm{~b}$. At this channel load, the gain is $50 \%$ with respect to ECRA-SC and $67 \%$ with respect to CRA. Interestingly, the normalized capacity for ECRA-MRC as well as for both ECRA-SC and CRA is relatively constant for heavy channel load i.e., $G>3$. In this way, the schemes appear to be robust against channel load fluctuations. On the other hand, the rate for which the maximum spectral efficiency $\xi^{*}$ (and so the normalized capacity) of the schemes is achieved lowers as the channel load increases; see Fig. 7b. Therefore the system would be required to adapt the rate in order to reach the best performance in terms of normalized capacity. Nevertheless, the adaptation of the rate remains quite limited in this channel load region, ranging from a maximum of 0.53 at $G=3$ to a minimum of 0.27 at $\mathrm{G}=6$ for ECRA-MRC. For limited channel load, all the schemes performs very close, with ALOHA being slightly the best option. This is due to the low collision probability and the benefit of double transmit power of ALOHA compared to CRA or ECRA since no replicas are sent.

In Fig. $7 b$, the rate corresponding to the maximum spectral efficiency for ECRA-MRC, ECRA-SC and CRA is shown. The maximum possible rate under this scenario, is also depicted with a solid line in the figure. For limited channel load, the maximum spectral efficiency is achieved when using the maximum rate allowed, supporting the fact that collisions of received packets are seldom and the spectral efficiency can be maximized pushing the rate as much as it is allowed. On the other hand, as soon as the channel load exceeds $G=0.3-0.4$, the maximum spectral efficiency is reached for rate values below the maximum one. In this way, the maximum spectral efficiency under moderate to high channel load conditions can be maximized taking a margin with respect to the maximum rate. This margin is helpful to counteract part of the collisions and at the same time does not reduces heavily the spectral efficiency. ${ }^{14}$

\section{CONCLusions}

In this paper, a novel frame- and slot-asynchronous RA decoding algorithm, named ECRA, has been presented. Motivated by the presence of $\mathcal{C}$-UCPs, ECRA tries to reduce their detrimental impact on the receiver's SIC procedure, applying combing techniques. ECRA exploits the presence of multiple instances of the same packet, in order to trigger the SIC procedure. In addition, ECRA tries to further reduce the interference, attempting to resolve partial collisions among packets, with the creation of a combined observation. The combined observation can be, either generated from the replicas sections

\footnotetext{
${ }^{14}$ Please note that the rate for ALOHA is not depicted in Fig. $7 \mathrm{~b}$ because it has a different degree $d$, and therefore the results are not comparable.
} 


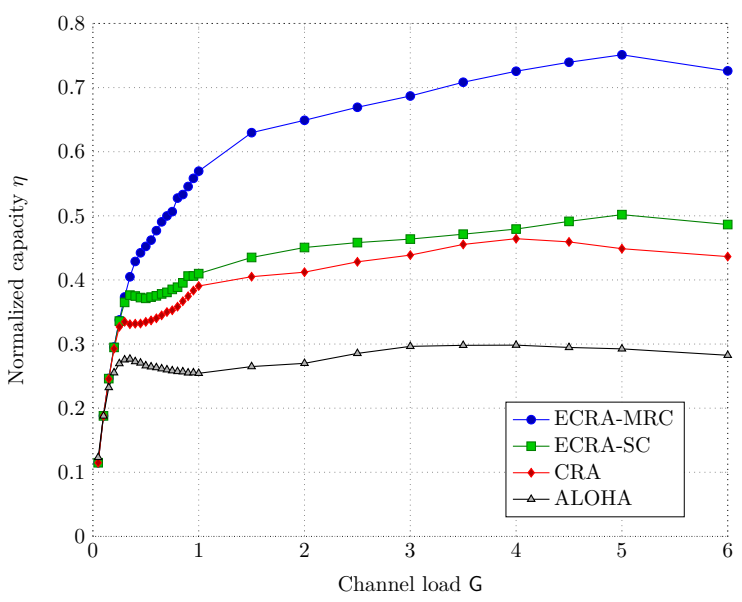

(a) Normalized capacity $\eta$ for ALOHA, CRA, ECRA-SC and ECRA-MRC with $\mathrm{P}_{g} / \mathrm{N}=6 \mathrm{~dB}$.

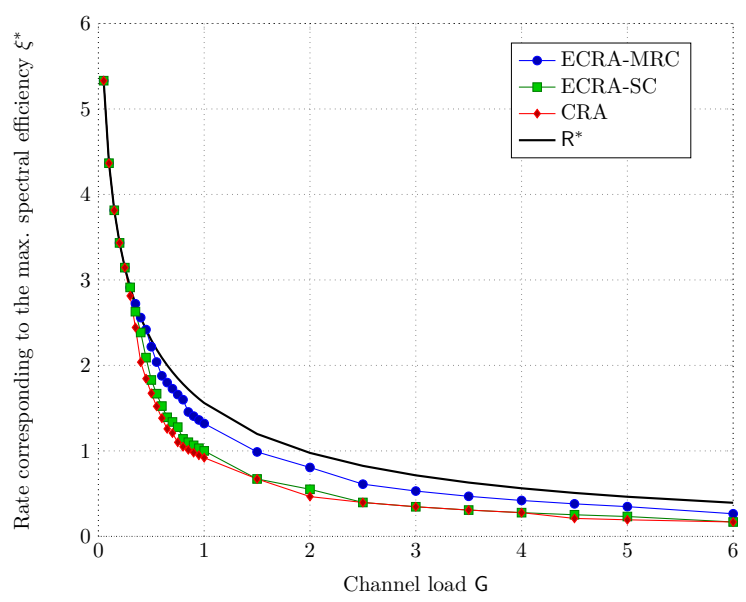

(b) Rate maximizing the spectral efficiency for CRA, ECRA-SC and ECRA-MRC with $\mathrm{P}_{g} / \mathrm{N}=6 \mathrm{~dB}$.

Fig. 7: Normalized capacity $\eta$ for ALOHA, CRA, ECRA-SC and ECRA-MRC with $\mathrm{P}_{g} / \mathrm{N}=6 \mathrm{~dB}$ and corresponding rate.

with the lowest level of interference, resorting to SC, or from the weighted combination of the replicas symbols of each user, resorting to MRC. An analytical approximation of the PLR, particularly tight for low to moderate channel load, is derived, considering only the $\mathcal{C}$-UCP involving two users. A comprehensive framework, with several metrics, is exploited for comparing both asynchronous and slot synchronous schemes, in the presence of channel coding. Finally, an investigation on the performance of ECRA under average power constraint, is performed. Numerical simulations show that, ECRA in both its variants, largely outperforms CRA, for all the considered scenarios, in terms of both throughput and PLR. Throughput exceeding 1 packet per packet duration and PLR below $10^{-4}$ for channel load up to $\mathrm{G}=0.6$ are achieved by ECRA-MRC. Gains of up to $100 \%$ in the maximum throughput, w.r.t. CRA, can be expected adopting ECRA-MRC while ECRA-SC has an improvement of $21 \%$ w.r.t. CRA. For a properly selected rate, ECRA-MRC is also able to outperform CRDSA with the same number of replicas. Finally, ECRA-MRC shows remarkable performance gains in terms of normalized capacity w.r.t. the other asynchronous RA schemes, reaching up to $75 \%$ the MAC channel capacity.

\section{ACKNOWLEDGMENT}

The authors would like to thank Dr. Gianlugi Liva, German Aerospace Center (DLR), for the useful discussions.

\section{REFERENCES}

[1] N. Abramson, "Multiple Access in Wireless Digital Networks," Proc. IEEE, vol. 82, no. 9, pp. 1360-1370, Sep. 1994.

[2] C. Kissling, "Performance Enhancements for Asynchronous Random Access Protocols over Satellite," in Proc. IEEE Int. Conf. on Commun. (ICC), Kyoto, Japan, Jun. 2011, pp. 1-6.

[3] N. Abramson, "The ALOHA system: Another alternative for computer communications," in Proc. Fall Joint Computer Conf. (AFIPS), vol. 37, Montvale, N. J., Nov. 1970, pp. 281-285.

[4] L. G. Roberts, "ALOHA packet system with and without slots and capture," Proc. SIGCOMM Computer Commun. Review, vol. 5, no. 2, pp. 28-42, Apr. 1975.

[5] N. Abramson, "The Throughput of Packet Broadcasting Channels," IEEE Trans. Commun., vol. 25, no. 1, pp. 117-128, Jan. 1977.
[6] D. Pompili and I. Akyildiz, "Overview of Networking Protocols for Underwater Wireless Communications," IEEE Commun. Mag., vol. 47, pp. 97-102, Jan. 2009.

[7] Y. He and X. Wang, "An ALOHA-Based Improved Anti-Collision Algorithm for RFID Systems," IEEE Wireless Commun. Mag., vol. 20, no. 5, pp. 152-158, Oct. 2013.

[8] H. Menouar, F. Filali, and M. Lenardi, "A Survey and Qualitative Analysis of MAC Protocols for Vehicular Ad Hoc Networks," IEEE Wireless Commun. Mag., vol. 13, no. 5, pp. 30-35, Oct. 2006.

[9] C. Stefanovic and P. Popovski, "ALOHA Random Access that Operates as a Rateless Code," IEEE Trans. Commun., vol. 61, no. 11, pp. 46534662, Nov. 2013.

[10] H. Peyravi, "Medium Access Control Protocols Performance in Satellite Communications," IEEE Commun. Mag., vol. 37, no. 3, pp. 62-71, Mar. 1999.

[11] E. Casini, R. De Gaudenzi, and O. del Rio Herrero, "Contention Resolution Diversity Slotted ALOHA (CRDSA): An Enhanced Random Access Scheme for Satellite Access Packet Networks," IEEE Trans. Wireless Commun., vol. 6, no. 4, pp. 1408-1419, Apr. 2007.

[12] G. Choudhury and S. Rappaport, "Diversity ALOHA - A Random Access Scheme for Satellite Communications," IEEE Trans. Commun., vol. 31, no. 3, pp. 450-457, Mar. 1983.

[13] L. Kleinrock, Queueing Systems - Volume II: Computer Applications, J. W. . Sons, Ed. Wiley Interscience, 1976.

[14] O. del Rio Herrero and R. de Gaudenzi, "Generalized Analytical Framework for the Performance Assessment of Slotted Random Access Protocols," IEEE Trans. Wireless Commun., vol. 13, no. 2, pp. 809-821, Feb. 2014

[15] C. Kissling, "On the stability of Contention Resolution Diversity Slotted ALOHA (CRDSA)," in Proc. IEEE Global Commun. Conf. (GLOBECOM), Houston, TX, USA, Dec. 2011, pp. 1-6.

[16] G. Liva, "Graph-Based Analysis and Optimization of Contention Resolution Diversity Slotted ALOHA," IEEE Trans. Commun., vol. 59, no. 2, pp. 477-487, Feb. 2011.

[17] E. Paolini, G. Liva, and M. Chiani, "Coded Slotted ALOHA: A GraphBased Method for Uncoordinated Multiple Access," IEEE Trans. Inform. Theory, vol. 61, no. 12, pp. 6815-6832, Oct. 2015.

[18] K. R. Narayanan and H. D. Pfister, "Iterative Collision Resolution for Slotted ALOHA: An Optimal Uncoordinated Transmission Policy," in Proc. 7th Int. Symp. on Turbo Codes and Iterative Information Processing (ISTC), Gothenburg, Sweden, Aug. 2012, pp. 136-139.

[19] L. Tong and V. Naware, "Signal Processing in Random Access," IEEE Signal Processing Mag., vol. 21, no. 5, pp. 29-39, Sep. 2004.

[20] H.-C. Bui, K. Zidane, J. Lacan, and M.-L. Boucheret, "A Multi-Replica Decoding Technique for Contention Resolution Diversity Slotted Aloha," in Proc. 82nd IEEE Vehicular Tec. Conf. (VTC-Fall), Boston, MA, Sep. 2015, pp. 1-6.

[21] M. Ghanbarinejad and C. Schlegel, "Irregular Repetition Slotted ALOHA with Multiuser Detection," in Proc. 10th Annual Conf. on 
Wireless On-demand Network Systems and Services (WONS), Banff, AB, Mar. 2013, pp. 201-205.

[22] C. Stefanovic, P. Popovski, and D. Vukobratovic, "Frameless ALOHA Protocol for Wireless Networks," IEEE Commun. Lett., vol. 16, no. 12 , pp. 2087-2090, Oct. 2012.

[23] A. Munari, M. Heindlmaier, G. Liva, and M. Berioli, "The Throughput of Slotted ALOHA with Diversity," in Proc. 51st Annual Allerton Conf. on Commun., Control, and Computing, Monticello, IL, USA, Oct. 2013, pp. 698-706.

[24] D. Jakovetic, D. Bajovic, D. Vukobratovic, and V. Crnojevic, "Cooperative Slotted Aloha for Multi-Base Station Systems," IEEE Trans. Commun., vol. 63, no. 4, pp. 1443-1456, Apr. 2015.

[25] M. Ivanov, F. Brännström, G. Graell i Amat, and P. Popovski, "All-to-all Broadcast for Vehicular Networks Based on Coded Slotted ALOHA," in Proc. IEEE Int. Conf. on Commun. (ICC) MASSAP Workshop, London, UK, Jun. 2015, pp. 2046-2050

[26] O. del Rio Herrero and R. De Gaudenzi, "High Efficiency Satellite Multiple Access Scheme for Machine-to-Machine Communications," IEEE Trans. Aerosp. Electron. Syst., vol. 48, no. 4, pp. 2961-2989, Oct. 2012.

[27] G. Gallinaro, F. Di Cecca, M.-A. Marchitti, R. De Gaudenzi, and O. del Rio Herrero, "Enhanced spread spectrum ALOHA system level performance assessment," Int. J. Satell. Commun. Network., vol. 32, no. 6, pp. 485-503, Nov.-Dec. 2014.

[28] R. De Gaudenzi, O. del Rio Herrero, G. Acar, and E. G. Barrabes, "Asynchronous Contention Resolution Diversity ALOHA: Making CRDSA Truly Asynchronous," IEEE Trans. Wireless Commun., vol. 13, no. 11, pp. 6193-6206, Nov. 2014.

[29] D. G. Brennan, "Linear Diversity Combining Techniques," Proc. IRE, vol. 47, no. 6, pp. 1075-1102, Jun. 1959.

[30] W. C. Jakes, Microwave Mobile Communication. Wiley-IEEE Press, 1974.

[31] F. Clazzer and C. Kissling, "Enhanced Contention Resolution ALOHA - ECRA," in Proc. 9th Int. ITG Conf. on Systems, Commun. and Coding (SCC), Munich, Germany, Jan. 2013, pp. 1-6.

[32] U. Mengali and A. N. D'Andrea, Synchronization Techniques for Digital Receivers, R. W. Lucky, Ed. Springer Science+Business Media, 1997.

[33] R. J. McEliece and W. E. Stark, "Channels with Block Interference," IEEE Trans. Inform. Theory, vol. IT-30, no. 1, pp. 44-53, Jan. 1984.

[34] T. M. Cover and J. A. Thomas, Elements of Information Theory, 2nd edition. John Wiley \& Sons, 2006.

[35] G. Thomas, "Capacity of the Wireless Packet Collision Channel With Feedback," IEEE Trans. Inform. Theory, vol. 46, no. 3, pp. 1141-1144, May 2000.

[36] S. Ghez, S. Verdu', and S. C. Schwartz, "Stability Properties of Slotted Aloha with Multipacket Reception Capability," IEEE Trans. Automat. Contr., vol. 33, no. 7, pp. 640-649, Jul. 1988.

[37] M. Zorzi and R. R. Rao, "Capture and Retransmission Control in Mobile Radio," IEEE J. Select. Areas Commun., vol. 12, no. 8, pp. 1289-1298, Oct. 1994.

[38] A. Meloni, M. Murroni, C. Kissling, and M. Berioli, "Sliding WindowBased Contention Resolution Diversity Slotted ALOHA," in Proc. IEEE Global Commun. Conf. (GLOBECOM), Anaheim, CA, USA, Dec. 2012, pp. 3305-3310.

[39] J. H. Winters, "Optimum Combining in Digital Mobile Radio with Cochannel Interference," IEEE J. Select. Areas Commun., vol. SAC-2, no. 4, pp. 528-539, Jul. July 1984.

[40] F. Clazzer and C. Kissling, "Optimum Header Positioning in Successive Interference Cancellation (SIC) based ALOHA," in Proc. IEEE Int. Conf. on Commun. (ICC), Budapest, Hungary, Jun. 2013, pp. 28692874.

[41] F. Clazzer, F. Lázaro, G. Liva, and M. Marchese, "Detection and Combining Techniques for Asynchronous Random Access with Time Diversity," in Proc. 11th Int. ITG Conf. on Systems, Commun. and Coding (SCC), Hamburg, Germany, Feb. 2017, pp. 1-6.

[42] S. Gollakota and D. Katabi, "ZigZag Decoding: Combating Hidden Terminals in Wireless Networks," in Proc. Int. Conf. of Special Interest Group on Data Commun. (SIGCOMM), vol. 38, no. 4, Oct. 2008, pp. $159-170$.

[43] A. S. Tehrani, A. G. Dimakis, and M. J. Neely, "SigSag: Iterative Detection through Soft Message-Passing," IEEE J. Select. Topics Signal Processing, vol. 5, no. 8, pp. 1512-1523, Sep. 2011.

[44] M. Ivanov, F. Brännström, A. Graell i Amat, and P. Popovski, "Error Floor Analysis of Coded Slotted ALOHA Over Packet Erasure Channels," IEEE Commun. Lett., vol. 19, no. 3, pp. 419-422, Mar. 2015.
[45] E. Sandgren, A. Graell i Amat, and F. Brännström, "On Frame Asynchronous Coded Slotted ALOHA: Asymptotic, Finite Length, adn Delay Analysis," IEEE Trans. Commun., vol. 65, no. 2, pp. 691-703, Feb. 2017.

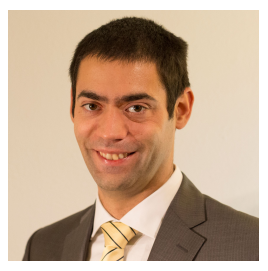

Federico Clazzer (S'11) was born in Genoa, Italy in 1987. He received the M.S. and the Ph.D. degrees in electrical engineering from the University of Genoa (Italy) in 2012 and 2017, respectively. His main research interests include satellite communication systems, random access techniques and signal processing algorithms. Since 2012 he is with the Institute of Communications and Navigation of the German Aerospace Center (DLR). During the past years he has been involved in several national and international projects on advanced medium access and random access techniques. In 2014, 2015 and 2016 he has been a frequent visitor at the Institute of Network Coding (INC), the Chinese University of Hong Kong. Aim of the collaboration has been the development of network coding techniques for the satellite communication scenario. Dr. Clazzer is IEEE member and he serves IEEE as reviewer for Transactions, Journals and Conferences as well as a technical program committee member. In 2017 he has been appointed as Exemplary Reviewer for IEEE Transactions on Communications.

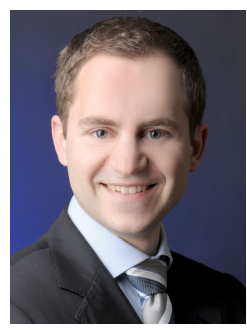

Christian Kissling Prof. Dr. Christian Kissling was born in Berchtesgaden, Germany in 1980. He received his Dipl.-Ing. degree in Electrical Engineering and Information Technology from the Technische Universität München (TUM) in 2005 and his Ph.D. degree with magna cum laude in electrical engineering from the Technische Universität München (TUM) in 2014. In 2005 he joined the German Aerospace Center (DLR), Oberpfaffenhofen as a scientific researcher and has been working there at the institute of communication and navigation in the area of satellite and aeronautical communication. In 2014 he joined the company Zodiac Aerospace / Zodiac Inflight Innovations and led a product development of aeronautical 3GPP server units as part of in-flightentertainment. In 2016 he then became Professor at the University of Applied Sciences Munich in the area of electrical and computer engineering. His current research interests include wireless communication technologies as well as embedded microcontroller systems for smart homes and ambient assisted living.

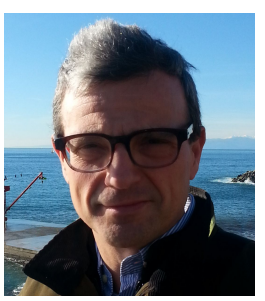

Mario Marchese (S'94-M'97-SM'04) was born in Genoa, Italy in 1967. He got his Laurea degree cum laude at the University of Genoa, Italy in 1992, and his Ph.D. (Italian Dottorato di Ricerca) degree in Telecommunications at the University of Genoa in 1997. From 1999 to January 2005, he worked with the Italian Consortium of Telecommunications (CNIT), by the University of Genoa Research Unit, where he was Head of Research. From February 2005 to January 2016 he was Associate Professor at the University of Genoa. Since February 2016 he has been Full Professor at the University of Genoa. He was the Chair of the IEEE Satellite and Space Communications Technical Committee from 2006 to 2008. He is Winner of the IEEE ComSoc Award 2008 Satellite Communications Distinguished Service Award in recognition of significant professional standing and contributions in the field of satellite communications technology. $\mathrm{He}$ is the author of the book Quality of Service over Heterogeneous Networks, John Wiley \& Sons, Chichester, 2007, and author/co-author of more than 290 scientific works, including international magazines, international conferences and book chapters. His main research activity concerns: Networking, Quality of Service over Heterogeneous Networks, Software Defined Networking, Satellite DTN and Nanosatellite Networks, Networking security. 